\title{
A 9-year Trend in the Prevalence of Allergic Disease Based on National Health Insurance Data
}

\author{
Byoungin Yoo, Yoonhyung Park, Kwanjun Park*, Hoseob Kim \\ Department of Preventive Medicine, Soonchunhyang University College of Medicine, Chunan, Korea
}

Objectives: To investigate trends in the prevalence of allergic disease over a 9-year period.

Methods: Using National Health Insurance Service (NHIS) data, the annual number of patients with allergic disease was obtained for each regional subdivisions (small cities, counties, and districts) from 2003 to 2011. Annual populations for each sub-region were obtained and used to calculate the standardized prevalence. To compare prevalence within the study period, data was standardized spatially and temporally. For standardization, demographic data was used to obtain the registered population and demographic structure for 2010, which was used to perform direct standardization of previous years. In addition, a geographic information system (GIS) was used to visualize prevalence for individual sub-regions, and allergic diseases were categorized into five groups according to prevalence.

Results: The nationwide outpatient prevalence of allergic rhinitis increased approximately 2.3 -fold, from $1.27 \%$ in 2003 to $2.97 \%$ in 2013, while inpatient prevalence also increased approximately 2.4-fold,. The outpatient prevalence of asthma increased 1.2-fold, and inpatient prevalence increased 1.3-fold. The outpatient prevalence of atopic dermatitis decreased approximately $12 \%$, and inpatient prevalence decreased $5 \%$.

Conclusions: There was a large difference between prevalence estimated from actual treatment data and prevalence based on patients' self-reported data, particularly for allergic rhinitis. Prevalence must continually be calculated and trends should be analyzed for the efficient management of allergic diseases. To this end, prevalence studies using NHIS claims data may be useful.

Key words: Asthma, Allergic rhinitis, Atopic dermatitis, Prevalence

Received: February 17, 2015 Accepted: October 26, 2015

Corresponding author: Yoonhyung Park, MD, PhD

31 Suncheonhyang 6-gil, Dongnam-gu, Cheonan 31151, Korea

Tel: +82-41-570-2406, Fax: +82-41-575-2406

E-mail: parky@sch.ac.kr

*Current affiliation: Division of Infectious Disease Control, Korea Centers For Disease Control and Prevention, Cheongju, Korea

This is an Open Access article distributed under the terms of the Creative Commons Attribution Non-Commercial License (http://creativecommons.org/licenses/by$\mathrm{nc} / 3.0 /$ ) which permits unrestricted non-commercial use, distribution, and reproduction in any medium, provided the original work is properly cited.

\section{INTRODUCTION}

Globally, three million individuals live with asthma and four million individuals live with allergic rhinitis [1,2]. Atopic dermatitis, allergic rhinitis, and asthma are mutually connected by the concept of the 'atopic march', and generally atopic dermatitis presents as the first symptom in the atopic march, developing into allergic rhinitis and asthma during the first few years of life [3].

There have been numerous studies investigating the global prevalence of, and risk factors for, asthma and other allergic diseases. The International Study of Asthma and Allergies in 
Childhood study investigated prevalence and risk factors for asthma and allergic diseases in infants and children worldwide [4]. The study proceeded in three phases to investigate changes in prevalence. The first phase investigated the prevalence of allergic diseases and of related symptoms; the second phase investigated risk factors affecting the prevalence of asthma; and the third phase investigated prevalence at least five years after the previous stages [5]. In South Korea (hereafter Korea), same methodological studies were conducted in 1995 [6], and 2000 [7]. Following the study in 2000, an additional epidemiological survey was conducted on a nationwide scale in 2010 [8-10]. According to the results of the Korean study, although the prevalence of asthma remained constant, the prevalence of allergic conjunctivitis and atopic dermatitis showed increasing trends, and the prevalence of allergic diseases was high when compared with global prevalence [10].

Korean studies measuring the prevalence of allergic diseases nationwide include the Korea National Health and Nutrition Examination Survey (KNHANES) and the Korea Youth Risk Behavior Web-based Survey (KYRBWS). The KNHANES surveys a probabilistic sample representing the national population, in order to ascertain national trends in health and nutrition [11]. The KYRBWS is an annual, anonymous, web-based survey of youths from 12 to 17 years old to investigate social factors of health and the prevalence of several diseases and injuries.

Another method for investigating the prevalence of allergic diseases is to use data from the National Health Insurance Service (NHIS). In particular, for Korea and Taiwan, the entire population is registered for medical insurance, and almost all medical facilities contract with NHIS, making it possible to analyze the collected data and estimate the prevalence of certain diseases [12-14]. We ascertained the number of inpatients and outpatients nationwide for allergic diseases using medical insurance claims data for allergic diseases from 2003 to 2011.

Small area variation has long been a topic of interest in public health studies [15], and in Korea as well, it is a widely-used method of analysis to investigate differences in health care utilization and interregional variations in prevalence [16-18]. In addition to small-area variation analysis, we performed an analysis using geographic information system (GIS) to visually compare differences in prevalence between years and sub-regions (small cities, counties, and districts).

\section{METHODS}

\section{Research Data}

Medical insurance claims data was used for present study. Medical insurance claims data can be categorized into claims data, which is related to claims by medical institutions, and certification data, which is related to recipients of medical services. Claims data related to claims made by medical institutions for medical services, and thus contains disease information, including treatments and the time of treatment. Conversely, certification data contains information about recipients of medical services, such as their place of residence and whether they are able to receive medical insurance or medical aid. Generally, these two types of data are collectively called medical insurance claims data.

Variables required for data processing included personal ID, place of residence, treatment starting date, disease code, form of treatment, major/minor diseases, and number of days in the hospital.

\section{Data Processing and the Definition of Prevalence}

In order to ascertain prevalence, the data was processed per individual, rather than per claim, on the basis of the following three assumptions:

First, patients with an allergic disease visit a doctor to receive treatment. NHIS data can be used to obtain data for all patients receiving treatment. Patients who experience symptoms (coughing, rhinorrhea, itching, etc.) but treat themselves at home without visiting a physician are not included in the data for allergic diseases.

Second, because allergic disease cannot be cured and continue to occur periodically, in the case of repeat visits during the 9-year study period, each patient was only included once in the annual prevalence.

Third, prevalence is the actual number of patients receiving treatment for allergic disease each year, divided by the estimated population for that year.

Statistical analysis was performed using SAS version 9.3 (SAS Institute Inc., Cary, NC, USA).

\section{Small Area Variation Analysis}

The number of patients for each sub-region (small cities, counties, and districts), standardized relative to the population structure in 2010, was divided by the population to calculate that sub-region's prevalence in the years from 2003 to 2011. 
This sub-regional prevalence was used to produce a small area variation analysis for each major region (large cities and provinces). The mean, standard deviation (SD), minimum, and maximum prevalence for allergic disease was calculated for each major region, and inter-regional variation was analyzed on the basis of these statistics. The extremal quotient (EQ), which is the ratio of the maximum and minimum values, and the coefficient of variation (CV), which is the ratio of the mean and SD, were used as common statistics to show variation.

\section{Use of Geographic Information System}

We categorized each of allergic disease into 5 groups by prevalence of allergic disease in sub-regional level. Because the prevalence was different for each allergic disease, individual diseases were categorized differently. A GIS was used to make visual comparisons between prevalence in different sub-regions.

\section{RESULTS}

\section{Allergic Rhinitis Prevalence}

The nationwide outpatient prevalence for allergic rhinitis increased approximately 2.3 -fold, from $1.27 \%$ in 2003 to $2.97 \%$ in 2011 (Supplemental Table 1). The inpatient prevalence also increased 2.4 -fold, from $0.05 \%$ in 2003 to $0.12 \%$ in 2011 . The annual trends for allergic rhinitis outpatient prevalence from 2003 to 2011 by age group are summarized in Table 1. Looking at trends at the level of major regions (large cities and provinces), the number of patients using outpatient services for aller- gic rhinitis increased in 2011 compared to 2003 for all cities and provinces. In Supplemental Figure 1, GIS is used to display the distribution of outpatient prevalence for allergic rhinitis among sub-regions (small cities, counties, and districts) in 2003 and 2011. The number of inpatients due to allergic rhinitis increased in 2011 compared to 2003 for all major regions. In addition, for both inpatients and outpatients, the number of sub-regions in the highest prevalence category increased in 2011 compared to 2003.

\section{Asthma Prevalence}

Nationwide, outpatient prevalence for asthma increased approximately 1.2 -fold, from $6.08 \%$ in 2003 to $7.35 \%$ in 2011 . Prevalence peaked at $7.38 \%$ in 2009 , after which it decreased slightly to $7.35 \%$ in 2011 . Inpatient prevalence increased approximately 1.3 -fold, from $0.20 \%$ in 2003 to $0.26 \%$ in 2011 . Inpatient prevalence decreased temporarily in 2006 and 2008, but it increased subsequently. Trends in the outpatient prevalence for asthma between 2003 and 2011 by age group are summarized in Table 2. An examination of the results by major region shows that the number of patients using outpatient services for asthma increased in 2011 compared to 2003 for all regions. The number of inpatients for asthma also increased in 2011 compared to 2003 for all major regions excluding Gyeonggi-do. In Supplemental Figure 2, GIS is used to display the distribution of outpatient prevalence for asthma among sub-regions in 2003 and 2011. For outpatients, the number of sub-regions in the highest and lowest prevalence categories both decreased.

Table 1. Annual number of outpatients with allergic rhinitis

\begin{tabular}{lrrrrrrrrr}
\hline Age $(\mathbf{y})$ & $\mathbf{2 0 0 3}$ & $\mathbf{2 0 0 4}$ & $\mathbf{2 0 0 5}$ & $\mathbf{2 0 0 6}$ & $\mathbf{2 0 0 7}$ & $\mathbf{2 0 0 8}$ & $\mathbf{2 0 0 9}$ & $\mathbf{2 0 1 0}$ & $\mathbf{2 0 1 1}$ \\
\hline Total & 609489 & 710500 & 796233 & 870219 & 980011 & 1122282 & 1389808 & 1407450 & 1478788 \\
$1-6$ & 193458 & 206619 & 214307 & 225266 & 251843 & 280434 & 310978 & 327214 & 346318 \\
$7-12$ & 83797 & 101501 & 115036 & 128177 & 140755 & 152568 & 194016 & 186821 & 189162 \\
$13-18$ & 28855 & 37013 & 45293 & 54107 & 59606 & 68974 & 111932 & 100925 & 99332 \\
$19-64$ & 261289 & 311637 & 360089 & 390883 & 440645 & 511693 & 637625 & 647412 & 689341 \\
$\geq 65$ & 42090 & 53730 & 61508 & 71786 & 87162 & 108613 & 135257 & 145078 & 154635 \\
\hline
\end{tabular}

Table 2. Annual number of outpatients with asthma

\begin{tabular}{lrrrrrrrrr}
\hline Age $(\mathbf{y})$ & $\mathbf{2 0 0 3}$ & $\mathbf{2 0 0 4}$ & $\mathbf{2 0 0 5}$ & $\mathbf{2 0 0 6}$ & $\mathbf{2 0 0 7}$ & $\mathbf{2 0 0 8}$ & $\mathbf{2 0 0 9}$ & $\mathbf{2 0 1 0}$ & $\mathbf{2 0 1 1}$ \\
\hline Total & 2907912 & 3072900 & 3336362 & 3424936 & 3447121 & 3504679 & 3628251 & 3628949 & 3659586 \\
$1-6$ & 1126416 & 1121026 & 1159887 & 1163408 & 1148121 & 1115568 & 1112876 & 1148654 & 1165609 \\
$7-12$ & 348385 & 393998 & 442402 & 460711 & 438424 & 419136 & 458697 & 430882 & 438970 \\
$13-18$ & 77425 & 94301 & 113612 & 131707 & 124393 & 131543 & 177944 & 168833 & 152940 \\
$19-64$ & 1004722 & 1084852 & 1216443 & 1241500 & 1279667 & 1334210 & 1357230 & 1356877 & 1362471 \\
$\geq 65$ & 350964 & 378723 & 404018 & 427610 & 456516 & 504222 & 521504 & 523703 & 539596 \\
\hline
\end{tabular}


Table 3. Annual outpatient prevalence for atopic dermatitis

\begin{tabular}{lrrrrrrrrr}
\hline Age $(\mathbf{y})$ & $\mathbf{2 0 0 3}$ & $\mathbf{2 0 0 4}$ & $\mathbf{2 0 0 5}$ & $\mathbf{2 0 0 6}$ & $\mathbf{2 0 0 7}$ & $\mathbf{2 0 0 8}$ & $\mathbf{2 0 0 9}$ & $\mathbf{2 0 1 0}$ & $\mathbf{2 0 1 1}$ \\
\hline Total & 1620224 & 1655822 & 1636140 & 1538627 & 1552267 & 1569477 & 1516332 & 1516621 & 1497322 \\
$1-6$ & 834616 & 806648 & 757500 & 687028 & 685677 & 689379 & 644408 & 618247 & 608141 \\
$7-12$ & 280514 & 292112 & 288294 & 268772 & 264732 & 252154 & 237191 & 232825 & 216291 \\
$13-18$ & 106821 & 116727 & 124861 & 127090 & 131471 & 132127 & 131580 & 136562 & 133535 \\
$19-64$ & 360866 & 397412 & 419039 & 407813 & 415762 & 431600 & 433373 & 453585 & 460694 \\
$\geq 65$ & 37407 & 42923 & 46446 & 47924 & 54625 & 64217 & 69780 & 75402 & 78661 \\
\hline
\end{tabular}

For inpatients, the number of sub-regions in the lowest prevalence category decreased, and the number of regions in the highest prevalence category increased.

\section{Nationwide Atopic Dermatitis Prevalence}

The nationwide outpatient prevalence for atopic dermatitis decreased approximately $12 \%$, from $3.39 \%$ in 2003 to $3.01 \%$ in 2011. The prevalence fluctuated throughout the study period, but has shown a continuously decreasing pattern since 2008 . The outpatient prevalence also decreased approximately $5 \%$, from $0.0024 \%$ in 2003 to $0.0023 \%$ in 2011 . Similar to the number of outpatients, the inpatient prevalence has also shown a continuously decreasing trend since recording a prevalence of $0.0033 \%$ in 2006 . Trends in atopic dermatitis outpatient prevalence for different age groups from 2003 to 2011 are summarized in Table 3. An examination of major regions shows that the number of patients using outpatient services for atopic dermatitis decreased in 2011 compared to 2003 for all regions. In Supplementarl Figure 3, the geographic distribution of outpatient and inpatient prevalence for atopic dermatitis in 2003 and 2011 is displayed for sub-regions using GIS. For outpatients, the number of sub-regions in the highest prevalence category decreased, while the number of sub-regions in the lowest prevalence category increased. For inpatients, the number of sub-regions in the highest prevalence category was similar for 2003 and 2011, but the location of those sub-regions shifted.

\section{Small Area Variation Analysis Results}

For each disease and year, the regions with the largest variation statistics were identified. Allergic rhinitis outpatients showed the largest EQ and CV for Chungcheongnam-do and Gyeonggi-do in 2003, but in 2011, the largest EQ and CV were for Gyeongsangbuk-do and Gyeongsangnam-do. Allergic rhinitis inpatients showed the highest EQ in 2003 for Chungcheongnam-do and Gangwon-do, and the highest CV for
Chungcheongbuk-do and Gangwon-do. Meanwhile, in 2011, the regions with the highest EQ were Gyeongsangbuk-do and Gyeongsangnam-do, and the regions with the highest CV were Gyeongsangbuk-do and Jeollabuk-do. Asthma outpatients showed the highest EQ and CV in 2003 for Chungcheongnam-do and Chungcheongbuk-do. In 2011, EQ was highest for Gangwon-do and Gyeongsangbuk-do, while CV was highest for Gangwon-do and Chungcheongbuk-do. Asthma inpatients showed the highest EQ in 2003 for Jeollabuk-do and Chungcheongnam-do, while the highest CV was for Jeollabukdo and Chungcheongbuk-do. In 2011, the regions with the highest EQ were Jeollanam-do and Gyeonggi-do, while the regions with the highest $\mathrm{CV}$ were Gyeonggi-do and Ulsan. Atopic dermatitis outpatients in 2003 showed the highest EQ and CV for Chungcheongbuk-do and Gyeongsangbuk-do. In 2011, the regions with the highest EQ were Chungcheongbuk-do and Incheon, while the regions with the highest CV were Chungcheongbuk-do and Gangwon-do. For atopic dermatitis inpatients, cases occurred in all sub-regions in 2003 for Seoul, Busan, Daegu, Incheon, and Daejeon, and so the EQ analysis was only performed on these major regions. The regions with the highest EQ were Busan and Seoul, while the regions with the highest CV were Gangwon-do and Jeollanam-do. In 2011, the major regions with patients in all sub-regions were Seoul, Busan, Gwangju, Daejeon, and Ulsan, and so the EQ analysis was restricted to these regions. The regions with the highest EQ were Daejeon and Gwangju, while the regions with the highest CV were Gyeongsangnam-do and Gangwon-do. Due to the low number of regions included in the EQ analysis and the low number of patients, statistical interpretation of the results for atopic dermatitis inpatients is expected to be limited.

\section{DISCUSSION}

The present study investigated trends in atopic disease prevalence in the Korean population from 2003 to 2011, using 
treatment data from the NHIS. Studies that have investigated allergic diseases nationwide include the KYRBWS and the KNHANES. The results of these surveys showed the prevalence of specific diseases as a percentage of the sub-population, while the present study used the population. This means that there are limitations when comparing prevalence directly, so prevalence trends were compared instead. According to KYRBWS statistics [19], allergic rhinitis showed a prevalence of $24.5 \%$ in 2007 , and persistently increased to $26.3 \%$ in $2008,27.9 \%$ in $2009,32.2 \%$ in 2010 , and $33.9 \%$ in 2011 . In the present study, the outpatient prevalence of allergic rhinitis was $2.01 \%$ in 2007 , $2.29 \%$ in $2008,2.82 \%$ in $2009,2.85 \%$ in 2010 , and $2.97 \%$ in 2011 , which showed a large difference between the number of patients reporting symptoms in a questionnaire and the number of patients actually receiving treatment. Both surveys showed similar patterns of continually increasing prevalence. Asthma prevalence in KYRBWS was $8.5 \%$ in $2007,8.0 \%$ in 2008, $8.0 \%$ in $2009,9.0 \%$ in 2010 , and $9.2 \%$ in 2011 , showing a persistent increase. In the present study, after increasing from $7.09 \%$ in 2007 to $7.16 \%$ in 2008 , then $7.38 \%$ in 2009 , similar values were maintained. Atopic dermatitis prevalence in KYRBWS was $17.3 \%$ in $2007,18.5 \%$ in $2008,18.9 \%$ in $2009,22.0 \%$ in 2010, and $23.1 \%$ in 2011, showing a persistent increase. However, the present study showed a different pattern, with prevalence gradually decreasing from $3.20 \%$ in 2007 to $3.08 \%$ in $2008,3.07 \%$ in 2010 , and $3.08 \%$ in 2011.

The statistics from the KNHANES provide prevalence data for allergic diseases from 1998 to 2012 [20]. The survey has been performed every year since 2007, but before that, the survey was only conducted in 1998, 2001, and 2005. The KNHANES showed an allergic rhinitis prevalence of $2.7 \%$ in $2001,8.3 \%$ in $2005,11.7 \%$ in 2007 and in $2008,11.4 \%$ in $2009,14.9 \%$ in 2010 , and $13.6 \%$ in 2011 , showing a gradually increasing pattern that was consistent with the present study. For asthma prevalence, the KNHANES showed a gradually increasing pattern of $1.4 \%$ in $2001,2.1 \%$ in $2005,2.3 \%$ in $2007,2.8 \%$ in 2008 , $2.8 \%$ in $2009,3.1 \%$ in 2010 , and $3.1 \%$ in 2011 , and this is a similar pattern to the asthma outpatient prevalence in the present study, where a similar prevalence was maintained after 2009. The KNHANES only investigated atopic dermatitis prevalence from 2007, showing figures of $2.3 \%$ in $2007,3.1 \%$ in $2008,2.7 \%$ in $2009,3.1 \%$ in 2010 , and $3.0 \%$ in 2011. This differed from the outpatient prevalence for atopic dermatitis in the present study, which showed a small decrease after 2008. The outpatient and inpatient prevalence for allergic rhinitis, asthma, and atopic dermatitis from 2003 to 2011 are summarized in Supplemental Table 1.

The small area variation analysis results showed that the highest $\mathrm{EQ}$ and $\mathrm{CV}$ were usually in provinces, meaning that there are larger differences in prevalence within provinces than in major cities. Apart from asthma inpatients in 2011, the highest $E Q$ and $C V$ were in a province rather than a large city. Provinces generally showed larger variation in allergic disease prevalence than large cities.

In the majority of cases, the rankings for EQ and CV were similar for the same year and disease, but the regions with the highest EQ and CV in 2003 were usually different from 2011, demonstrating changes in the regional distribution of prevalence during the study period. Supplemental Table 2 summarizes the results to show the regions with the highest two EQ and $C V$ values for each year and each disease.

Although the present study analyzed whether there was a difference in prevalence between major regions and whether there was small area variation, it was not able to reveal why regional differences occur. Additional research will be required to find out why this difference was made.

Analysis of insurance claims data is known to provide data for establishing public health policies at less cost and effort than randomized clinical trials [21]. However, in studies using insurance claims data, problems with internal validity, construct validity, and external validity can arise [21].

If there is a discrepancy between the actual disease and the claims code, this causes problems with internal validity. According to research on the accuracy of claims codes with respect to actual diseases in Korea, although there are differences depending on the disease, the accuracy is approximately $70 \%$ to $80 \%$ [22], and disease codes at higher level medical institutions were more accurate [23]. However, there have been no studies on the accuracy of disease codes in Korea for allergic diseases, and so there could be issues with internal validity due to inaccuracy of the claims codes.

Compared to a similar study that obtained prevalence for outpatients in 2005 and 2008 using treatment data from the NHIS, the present study shows differences in prevalence [14]. In previous study, prevalence of allergic rhinitis was $7.79 \%$ and $9.08 \%$ in 2005 and 2008 which was 2-3 fold higher than present study. For the outpatient prevalence of asthma, the previous study found values of $4.61 \%$ in 2005 and $4.57 \%$ in 2008 , which was $50 \%$ lower than present study. Meanwhile, the outpatient prevalence for atopic dermatitis in the previous study 
was $2.33 \%$ in 2005 and $2.18 \%$ in 2008 , which was $30 \%$ lower than present study. These differences in prevalence demonstrate the issue of construct validity, and appear to be the result of different definitions of patients with the disease.

Different studies reach different conclusions about the effect of area of residence on allergic disease. In one study dividing regions into urban, rural, and industrial regions, the residential environment did not have an effect on asthmatic symptoms [6], and in another study distinguishing between large cities, small-to-medium cities, and rural areas, there was a difference in the prevalence of symptoms according to the area of residence for some diseases [10]. In another study comparing prevalence in 16 major cities and provinces across the country, there were differences in prevalence between regions and also differences in the rate of prevalence increase, but statistical tests on interregional differences were not performed [14]. Additional research will be required in the causes of interregional differences in the prevalence of allergic diseases, through linkage with local climatic data and observed dust concentration data, as collected by the Korea Meteorological Administration [24].

The annual cost of management and treatment for allergic disease is high, and it is possible that the prevalence of allergic disease will gradually increase due to the effects of climate change. Continual calculation of prevalence and analysis of trends is required for the efficient management of allergic disease. Prevalence surveys using NHIS claims data can be a useful instrument for this purpose, since they do not have problems with representativeness and have relatively few spatial or temporal limitations.

\section{ACKNOWLEDGEMENTS}

This study was supported by the "Development of ClimateChange Health Impact Assessment and Adaptation Technologies" project (no. 412-111-002) which is granted by KEITI \& the Ministry of the Environment as an Eco-Innovation Project.

\section{CONFLICT OF INTEREST}

The authors have no conflicts of interest associated with the material presented in this paper.

\section{REFERENCES}

1. Zheng T, Yu J, Oh MH, Zhu Z. The atopic march: progression from atopic dermatitis to allergic rhinitis and asthma. Allergy Asthma Immunol Res 2011;3(2):67-73.

2. Bousquet J, Khaltaev NG, Cruz AA; World Health Organization. Global surveillance, prevention and control of chronic respiratory diseases: a comprehensive approach. Geneva: World Health Organization; 2007, p.14.

3. Spergel JM. From atopic dermatitis to asthma: the atopic march. Ann Allergy Asthma Immunol 2010;105(2):99-106.

4. Asher MI, Keil U, Anderson HR, Beasley R, Crane J, Martinez F, et al. International Study of Asthma and Allergies in Childhood (ISAAC): rationale and methods. Eur Respir J 1995;8(3): 483-491.

5. Björkstén B, Clayton T, Ellwood P, Stewart A, Strachan D; ISAAC Phase III Study Group. Worldwide time trends for symptoms of rhinitis and conjunctivitis: Phase III of the International Study of Asthma and Allergies in Childhood. Pediatr Allergy Immunol 2008;19(2):110-124.

6. Hong SJ, Lee MS, Sohn MH, Shim JY, Han YS, Park KS, et al. Self-reported prevalence and risk factors of asthma among Korean adolescents: 5-year follow-up study, 1995-2000. Clin Exp Allergy 2004;34(10):1556-1562.

7. Lee SI. Prevalence of childhood asthma in Korea: International Study of Asthma and Allergies in Childhood. Allergy Asthma Immunol Res 2010;2(2):61-64.

8. Suh M, Kim HH, Sohn MH, Kim KE, Kim C, Shin DC. Prevalence of allergic diseases among Korean school-age children: a nationwide cross-sectional questionnaire study. J Korean Med Sci 2011;26(3):332-338.

9. Oh JW, Kim KE, Pyun BY, Lee HR, Choung JT, Hong SJ, et al. Nationwide study for epidemiological change of atopic dermatitis in school aged children between 1995 and 2000 and kindergarten aged children in 2003 in Korea. Pediatr Allergy Respir Dis 2003;13(4):227-237 (Korean).

10. Ahn K, Kim J, Kwon HJ, Chae Y, Hahm MI, Lee KJ, et al. The prevalence of symptoms of asthma, allergic rhinoconjunctivitis, and eczema in Korean children: nationwide cross-sectional survey using complex sampling design. J Korean Med Assoc 2011;54(7):769-778 (Korean).

11. Jung JY, Kim JS, Choi HJ, Lee KY, Park TJ. Factors associated with ADL and IADL from the Third Korea National Health and Nutrition Examination Survey (KNHANES III), 2005. Korean J Fam Med 2009;30(8):598-609 (Korean).

12. Wu MP, Hsu YW, Weng SF, Ho CH, Wang JJ, Tong YC. Healthcareseeking prevalence of lower urinary tract symptoms among national health insurance enrollees in Taiwan, 2000-2009. 
Urology 2013;81(1):61-65.

13. Tseng $\mathrm{CH}$. Diabetes and non-Hodgkin's lymphoma: analyses of prevalence and annual incidence in 2005 using the National Health Insurance database in Taiwan. Ann Oncol 2012;23(1): 153-158.

14. Seong HU, Cho SD, Park SY, Yang JM, Lim DH, Kim JH, et al. Nationwide survey on the prevalence of allergic diseases according to region and age. Pediatr Allergy Respir Dis 2012;22(3): 224-231 (Korean).

15. Do YK. Research on geographic variations in health services utilization in the United States: a critical review and implications. Health Policy Manag 2007;17(1):94-124 (Korean).

16. Lee S, Cho W, Nam C, Kim S. Diagnosis-specific analysis of small area variations in hospital services. Korean J Health Policy Adm 1994;4(1):49-76 (Korean).

17. Kim DH. Analysis of small area variation of health behavior using 2008 Community Health Survey in Korea. Cheongju: Korea Centers for Disease Control and Prevention; 2010, p. 27 (Korean).

18. Kwon YC, Kim KH, Chang DM. A study on small area variations of hospital services utilization in some acute diseases: focused on gastric diseases and acute appendicitis. J Digit Convergence 2012;10(7):193-200 (Korean).
19. Ministry of Education; Ministry of Health and Welfare; Korea Centers for Disease Control and Prevention. Reports on the Korean Youth Risk Behavior Web-based Survey 2013 [cited 2015 Feb 20]. Available from:http://yhs.cdc.go.kr/(Koean).

20. Korea Centers for Disease Control and Prevention. Korea health statistics 2012: Korea National Health and Nutrition Examination Survey (KNHANESV-3) 2012 [cited 2015 Oct 26]. Available from: https://knhanes.cdc.go.kr/knhanes/index.do (Korean).

21. Motheral BR, Fairman KA. The use of claims databases for outcomes research: rationale, challenges, and strategies. Clin Ther 1997;19(2):346-366.

22. Kimm H, Yun JE, Lee SH, Jang Y, Jee SH. Validity of the diagnosis of acute myocardial infarction in Korean national medical health insurance claims data: the Korean heart study (1). Korean Circ J 2012;42(1):10-15.

23. Park JK, Kim KS, Kim CB, Lee TY, Lee KS, Lee DH, et al. The accuracy of ICD codes for cerebrovascular diseases in medical insurance claims. Korean J Prev Med 2000;33(1):76-82 (Korean).

24. Korea Meteorological Administration. Estimated level of dust (PM10) [cited 2015 Mar 21]. Available from: http://www.kma. go.kr/weather/asiandust/density.jsp (Korean). 
Supplemental Table 1. Prevalence (\%) of allergic disease from 2003 to 2011

\begin{tabular}{lccccccccc}
\hline & $\mathbf{2 0 0 3}$ & $\mathbf{2 0 0 4}$ & $\mathbf{2 0 0 5}$ & $\mathbf{2 0 0 6}$ & $\mathbf{2 0 0 7}$ & $\mathbf{2 0 0 8}$ & $\mathbf{2 0 0 9}$ & $\mathbf{2 0 1 0}$ & $\mathbf{2 0 1 1}$ \\
\hline Allergic rhinitis outpatients & 1.27 & 1.48 & 1.65 & 1.80 & 2.02 & 2.29 & 2.83 & 2.85 & 2.97 \\
Allergic rhinitis inpatients & 0.05 & 0.06 & 0.07 & 0.07 & 0.08 & 0.09 & 0.11 & 0.12 & 0.12 \\
Asthma outpatients & 6.08 & 6.40 & 6.93 & 7.08 & 7.09 & 7.16 & 7.38 & 7.34 & 7.35 \\
Asthma inpatients & 0.20 & 0.20 & 0.22 & 0.24 & 0.23 & 0.23 & 0.23 & 0.25 & 0.26 \\
Atopic dermatitis outpatients & 3.39 & 3.45 & 3.40 & 3.18 & 3.19 & 3.21 & 3.08 & 3.07 & 3.01 \\
Atopic dermatitis inpatients & 0.0024 & 0.0024 & 0.0025 & 0.0033 & 0.0028 & 0.0026 & 0.0027 & 0.0025 & 0.0023 \\
\hline
\end{tabular}

Supplemental Table 2. Major regions with high statistics for variation, by disease

\begin{tabular}{|c|c|c|c|c|}
\hline & $\begin{array}{l}\text { Region with } \\
\text { highest EQ }\end{array}$ & $\begin{array}{c}\text { Region with } \\
\text { 2nd highest E0 }\end{array}$ & $\begin{array}{l}\text { Region with } \\
\text { highest CV }\end{array}$ & $\begin{array}{l}\text { Region with } \\
\text { 2nd highest CV }\end{array}$ \\
\hline Allergic rhinitis outpatients (2003) & Chungcheongnam-do & Gyeonggi-do & Chungcheongnam-do & Gyeonggi-do \\
\hline Allergic rhinitis inpatients (2003) & Chungcheongnam-do & Gangwon-do & Chungcheongbuk-do & Gangwon-do \\
\hline Allergic rhinitis inpatients (2011) & Gyeongsangbuk-do & Gyeongsangnam-do & Gyeongsangbuk-do & Jeollabuk-do \\
\hline Asthma outpatients (2003) & Chungcheongnam-do & Chungcheongbuk-do & Chungcheongnam-do & Chungcheongbuk-do \\
\hline Asthma inpatients (2003) & Jeollabuk-do & Chungcheongnam-do & Jeollabuk-do & Gyeongsangbuk-do \\
\hline Asthma inpatients (2011) & Jeollanam-do & Gyeonggi-do & Gyeonggi-do & Ulsan \\
\hline Atopic dermatitis outpatients (2003) & Chungcheongbuk-do & Gyeongsangbuk-do & Chungcheongbuk-do & Gyeongsangbuk-do \\
\hline Atopic dermatitis outpatients (2011) & Chungcheongbuk-do & Incheon & Chungcheongbuk-do & Gangwon-do \\
\hline Atopic dermatitis inpatients (2003) & Seoul & Busan & Chungcheongbuk-do & Gangwon-do \\
\hline
\end{tabular}

EQ, extremal quotient; CV, coefficient of variation. 


\section{Journal of}

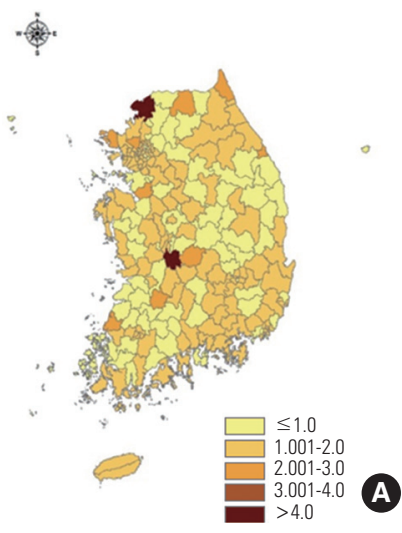

-察"

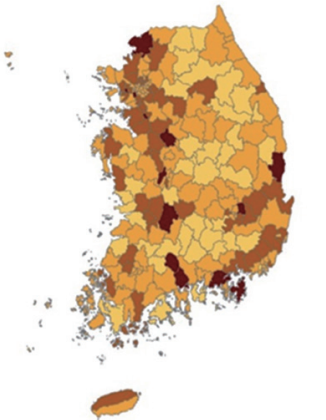

B

Supplemental Figure 1. Geographical distribution of annual outpatient prevalence for allergic rhinitis, by sub-region (A: 2003, B: 2011).
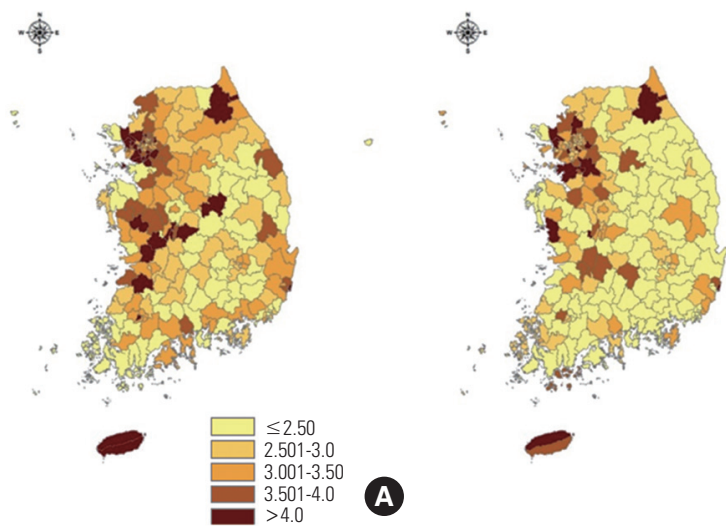

A

Supplemental Figure 3. Geographical distribution of annual outpatient prevalence for atopic dermatitis, by sub-region ( $A$ : 2003, B: 2011).
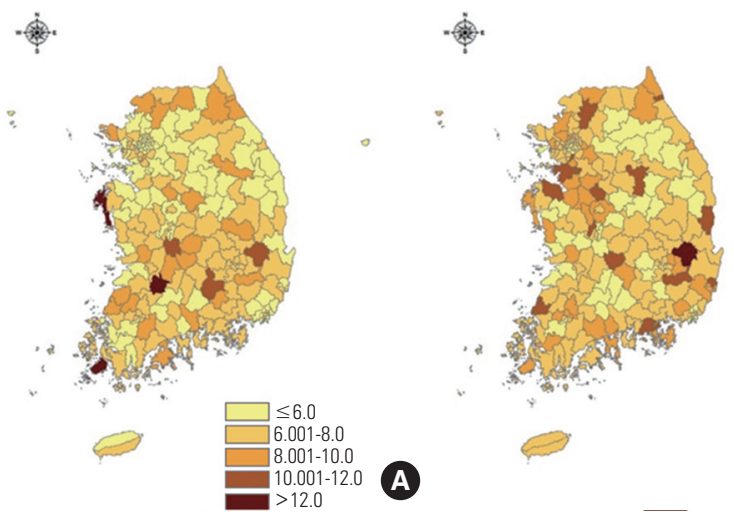

B

Supplemental Figure 2. Geographical distribution of annual outpatient prevalence for asthma, by sub-region (A: 2003, B: 2011). 